\title{
Advanced interatrial block and ischemic stroke
}

\author{
The Atherosclerosis Risk in Communities Study
}

Wesley T. O’Neal, MD, $\mathrm{MPH}$

Hooman Kamel, MD

Zhu-Ming Zhang, MD, $\mathrm{MPH}$

Lin Y. Chen, MD, MS

Alvaro Alonso, MD, PhD

Elsayed Z. Soliman, MD,

MS

Correspondence to

Dr. O'Neal:

wesley.oneal@emory.edu

Editorial, page 344

\section{ABSTRACT}

Objective: Given that recent reports have suggested left atrial disease to be an independent risk factor for ischemic stroke, we sought to examine if advanced interatrial block (alAB) is an independent stroke risk factor.

Methods: We examined the association between alAB and incident ischemic stroke in 14,716 participants (mean age $54 \pm 5.8$ years; $55 \%$ female; $26 \%$ black) from the Atherosclerosis Risk in Communities Study (ARIC). Cases of alAB were identified from digital ECGs recorded during the baseline ARIC visit (1987-1989) and the first 3 follow-up study visits (1990-1992, 1993-1995, and 1996-1998). Adjudicated ischemic stroke events were ascertained through December 31, 2010.

Results: There were 266 (1.8\%) participants who had evidence of alAB. Over a median follow-up of 22 years, 916 (6.2\%) ischemic stroke events were detected. The incidence rate (per 1,000 person-years) of ischemic stroke among those with alAB (incidence rate 8.05, 95\% confidence interval [CI] 5.7, 11.4) was more than twice the rate in those without al $\mathrm{AB}$ (incidence rate $3.14,95 \% \mathrm{Cl} 2.94,3.35$ ). In a multivariable Cox regression analysis adjusted for stroke risk factors and potential confounders, alAB was associated with an increased risk of ischemic stroke (hazard ratio 1.63, 95\% $\mathrm{Cl} 1.13,2.34$ ). The results were consistent across subgroups of participants stratified by age, sex, and race.

Conclusions: In the ARIC, aIAB was associated with incident ischemic stroke, which strengthens the hypothesis that left atrial disease should be considered an independent stroke risk factor.

Neurology ${ }^{\circledR}$ 2016;87:352-356

\section{GLOSSARY}

$\mathbf{A F}=$ atrial fibrillation; $\mathbf{a l A B}=$ advanced interatrial block; $\mathbf{A R I C}=$ Atherosclerosis Risk in Communities Study; $\mathbf{C l}=$ confidence interval; $\mathbf{H R}=$ hazard ratio; ICD-9 = International Classification of Diseases-9; PTFV $\mathbf{1}=$ P-wave terminal force in lead $\mathbf{V}_{\mathbf{1}}$.

Advanced interatrial block (aIAB) exists when a delay of conduction occurs over the Bachmann bundle and the left atrium is depolarized by retrograde activation via muscle connections near the coronary sinus. ${ }^{1}$ These abnormal properties often are observed among persons with risk factors for myocardial fibrosis and abnormal cardiac remodeling, suggesting that aIAB represents underlying left atrial disease.

Recent reports have suggested that ECG-detected left atrial abnormality, as measured by P-wave terminal force in lead $\mathrm{V}_{1}\left(\mathrm{PTFV}_{1}\right)$, is associated with an increased risk for ischemic stroke. ${ }^{2-4}$ This association was limited to nonlacunar infarcts and remained after accounting for incident atrial fibrillation (AF). ${ }^{4}$ These data suggest that left atrial disease possibly results in thromboembolism independent of diagnosed $\mathrm{AF}$ and that left atrial disease is an independent stroke risk factor. ${ }^{5}$

Given that abnormally increased $\mathrm{PTFV}_{1}$ and $\mathrm{aIAB}$ represent similar underlying pathologic changes in the left atrium, it is plausible that $\mathrm{aIAB}$ also represents a risk factor for ischemic stroke. Therefore, the purpose of this study was to examine the association between aIAB and incident ischemic stroke in the Atherosclerosis Risk in Communities Study (ARIC).

\footnotetext{
From the Department of Medicine (W.T.O.) and Epidemiological Cardiology Research Center, Department of Epidemiology and Prevention (Z.-M.Z., E.Z.S.), Wake Forest School of Medicine, Winston-Salem, NC; Department of Neurology (H.K.), Weill Cornell Medical College, New York, NY; and Department of Medicine, Cardiovascular Division (L.Y.C.), and Division of Epidemiology and Community Health, School of Public Health (A.A.), University of Minnesota, Minneapolis, MN. W.T.O. is currently with the Department of Medicine, Division of Cardiology, Emory University School of Medicine, Atlanta, GA; and A.A. is currently with the Department of Epidemiology, Rollins School of Public Health, Emory University, Atlanta, GA.

Go to Neurology.org for full disclosures. Funding information and disclosures deemed relevant by the authors, if any, are provided at the end of the article.
} 
METHODS Study design and population. A total of 15,792 community-dwelling men and women between 45 and 64 years of age were prospectively enrolled in ARIC from 4 field centers across the United States (Washington County, Maryland; Forsyth County, North Carolina; Jackson, Mississippi; suburban Minneapolis, Minnesota). Participants were recruited between 1987 and 1989 and returned for 4 follow-up examinations (1990 1992, 1993-1995, 1996-1998, and 2011-2013). Semiannual telephone calls are used to follow ARIC participants and ascertain study endpoints. Study endpoints are further ascertained from hospital discharge records.

Participants with prevalent stroke or AF at baseline were excluded from this analysis. Additionally, the few ARIC participants with race other than black or white were excluded, as were the small number of black participants from Washington County and Minneapolis.

Standard protocol approvals, registrations, and patient consents. This study was approved by the institutional review boards at Wake Forest University and each participating center. All participants provided written informed consent at the time of study enrollment.

Advanced interatrial block. Digital 12-lead ECGs were obtained at baseline and at subsequent study visits using MAC PC ECG machines (Marquette Electronics, Milwaukee, WI). All ECGs were transmitted electronically and inspected for technical errors at the Epidemiology Coordinating and Research Centre at the University of Alberta (Edmonton, Canada) during the initial phases of the study and at the Epidemiological Cardiology Research Center at the Wake Forest School of Medicine (Winston-Salem, NC) during later phases. aIAB was identified on the 12-lead ECG as the presence of $\mathrm{P}$-wave duration $\geq 120 \mathrm{~ms}$ and biphasic (positive negative) morphology in leads II, III, and aVF or biphasic (positive negative) morphology in leads III and aVF and notched P (positive positive) in lead II. ${ }^{1}$ Cases of aIAB were identified using baseline ECG data and also using ECGs from the first 3 follow-up study visits (1990-1992, 1993-1995, and 1996-1998).

Ischemic stroke. Our outcome of interest was definite and probable ischemic stroke. The ascertainment of stroke events in ARIC has been described previously. ${ }^{6}$ Potential cases were first identified during semiannual telephone calls and review of hospitalization records. Medical records were then reviewed and abstracted by a single trained nurse at a central location (University of Minnesota). Based on these records, the date and type of stroke was classified by a software algorithm that applied validated criteria from the National Survey of Stroke by the National Institute of Neurologic Disorders and Stroke. ${ }^{7}$ Strokes were subsequently classified as hemorrhagic, cardioembolic, or thrombotic. Each medical record was independently reviewed by a physician investigator and separately determined if a stroke occurred and classified each event as hemorrhagic or ischemic. Cases of disagreement between the software program and the independent physician investigator were adjudicated by a second physician.

Atrial fibrillation. Due to the potential for AF to explain the association between $\mathrm{aIAB}$ and ischemic stroke, we examined the association between both conditions with adjustment for incident $\mathrm{AF}$ as a time-dependent covariate. $\mathrm{AF}$ cases were identified from study ECGs, review of hospital discharge records, and death certificates. ${ }^{8}$ AF cases automatically detected during the study ECGs were visually confirmed by a cardiologist. ${ }^{9}$ Hospitalization data during follow-up were obtained from annual follow-up calls and local hospital surveillance. Additionally, hospital discharge diagnosis codes were collected by trained abstractors during follow-up and AF events were defined by ICD-9 codes 427.31 or 427.32 . $\mathrm{AF}$ events that were detected during hospitalizations for cardiac surgery were not included as these cases were considered to be transient. ${ }^{10}$

Baseline characteristics. Age, sex, and race were self-reported. Smoking was defined as the current or former use of cigarettes. Diabetes was defined as at least one of the following: fasting glucose level $\geq 126 \mathrm{mg} / \mathrm{dL}$, nonfasting glucose level $\geq 200$ $\mathrm{mg} / \mathrm{dL}$, self-reported physician diagnosis of diabetes, or the use of diabetes medications. Three systolic blood pressure measurements in the upright position after 5 minutes of rest were obtained from each participant using sphygmomanometers. The average of the last 2 blood pressure measurements was used as the final reading. Antihypertensive medication use was self-reported. Body mass index was defined as the weight in kilograms divided by the square of the height in meters. Low-density lipoprotein cholesterol levels were computed indirectly using cholesterol values assayed from serum samples. Heart failure was defined as present if participants reported taking heart failure medications or if participants met all 3 of the Gothenburg criteria. ${ }^{11}$ Coronary heart disease was defined by a self-reported history of physiciandiagnosed myocardial infarction, coronary artery bypass surgery, coronary angioplasty, or ECG evidence of myocardial infarction.

Statistical analysis. Baseline characteristics were examined by the presence of aIAB (prevalent and incident cases). Categorical variables were reported as frequency and percentage while continuous variables were recorded as mean $\pm S D$. The $\chi^{2}$ method was used to compare differences for categorical variables and the Student $t$ test was used for continuous variables. Follow-up time was defined as time between aIAB detection until ischemic stroke, death, loss to follow-up, or end of follow-up (December 31, 2010). For those with incident $\mathrm{aIAB}$, the time between the baseline visit and $\mathrm{aIAB}$ diagnosis was considered as non-aIAB follow-up. Kaplan-Meier estimates were used to examine the cumulative incidence of ischemic stroke by the presence of $\mathrm{aIAB}$ as a time-dependent variable. Cox regression was used to compute hazard ratios (HR) and $95 \%$ confidence intervals (CI) for the association between $\mathrm{aIAB}$ and ischemic stroke. Multivariable models were constructed as follows: model 1 adjusted for age, sex, and race; model 2 adjusted for model 1 covariates plus smoking, systolic blood pressure, diabetes, body mass index, low-density lipoprotein cholesterol, antihypertensive medication use, coronary heart disease, and heart failure; model 3 adjusted for model 2 covariates plus incident AF. The covariates included in the multivariable models were from the initial study visit, with the exception of time-dependent incident $\mathrm{aIAB}$ and incident $\mathrm{AF}$. Subgroup analyses were performed by age (dichotomized by the median age for study participants), sex, and race. Due to the known association between left atrial abnormality and ischemic stroke in ARIC, ${ }^{4}$ a sensitivity analysis was performed with adjustment for $\mathrm{PTFV}_{1}$ to determine if both markers independently predict stroke. Additionally, since clinically apparent $\mathrm{AF}$ is often not detected during acute stroke events, ${ }^{12}$ we included a separate analysis with adjustment for incident $\mathrm{AF}$ as a time-fixed covariate (i.e., incident cases of $\mathrm{AF}$ were modeled as having been present since baseline). Statistical significance was defined as $p<0.05$. SAS version 9.4 (Cary, NC) was used for all analyses.

RESULTS A total of 14,716 (mean age $54 \pm 5.8$ years; $55 \%$ female; $26 \%$ black) participants were 
Table 1 Baseline characteristics $(n=14,716)$

$\begin{array}{lllc}\text { Characteristics } & \begin{array}{l}\text { alAB } \\ (\mathbf{n}=266)\end{array} & \begin{array}{l}\text { No alAB } \\ (\mathbf{n}=14,450)\end{array} & \text { p Value } \\ \text { Age, y, mean (SD) } & 58(5.4) & 54(5.7) & <0.0001 \\ \text { Male, } \mathbf{n}(\%) & 169(64) & 6,411(44) & <0.0001 \\ \text { Black, } \mathbf{n}(\%) & 60(23) & 3,804(26) & 0.17 \\ \text { Smoking, } \mathbf{n}(\%) & 167(63) & 8,426(58) & 0.14 \\ \text { Diabetes, } \mathbf{n}(\%) & 47(18) & 1,606(11) & 0.0008 \\ \text { LDL cholesterol, mg/dL, mean (SD) } & 146(37) & 137(39) & 0.0007 \\ \text { Body mass index, kg/m², mean (SD) } & 30(5.3) & 28(5.3) & <0.0001 \\ \text { Systolic blood pressure, mm Hg, mean (SD) } & 130(20) & 121(19) & <0.0001 \\ \text { Antihypertensive medications, } \mathbf{n}(\%) & 153(58) & 4,260(29) & <0.0001 \\ \text { Coronary heart disease, } \mathbf{n} \text { (\%) } & 33(12) & 671(4.6) & <0.0001 \\ \text { Heart failure, } \mathbf{n}(\%) & 27(10) & 643(4.5) & <0.0001\end{array}$

Abbreviations: alAB = advanced interatrial block; $L D L=$ low-density lipoprotein. a Statistical significance for categorical data was tested using the $\chi^{2}$ procedure and continuous data was tested using Student $t$ test.

included in the final analytical sample. There were $266(1.8 \%)$ participants who had ECG evidence of aIAB. Of these, 70 were detected at the baseline examination and 196 were detected during the first 3 follow-up visits. Baseline characteristics stratified by the presence of aIAB are shown in table 1 .

Over a median follow-up of 22 years, $916(6.2 \%)$ ischemic stroke events were detected. The incidence rate (per 1,000 person-years) of ischemic stroke was higher among those with aIAB (incidence rate 8.05, $95 \%$ CI $5.7,11.4)$ compared with those without aIAB (incidence rate $3.14,95 \%$ CI 2.94, 3.35).

Figure Cumulative incidence of ischemic stroke by advanced interatrial block (alAB)

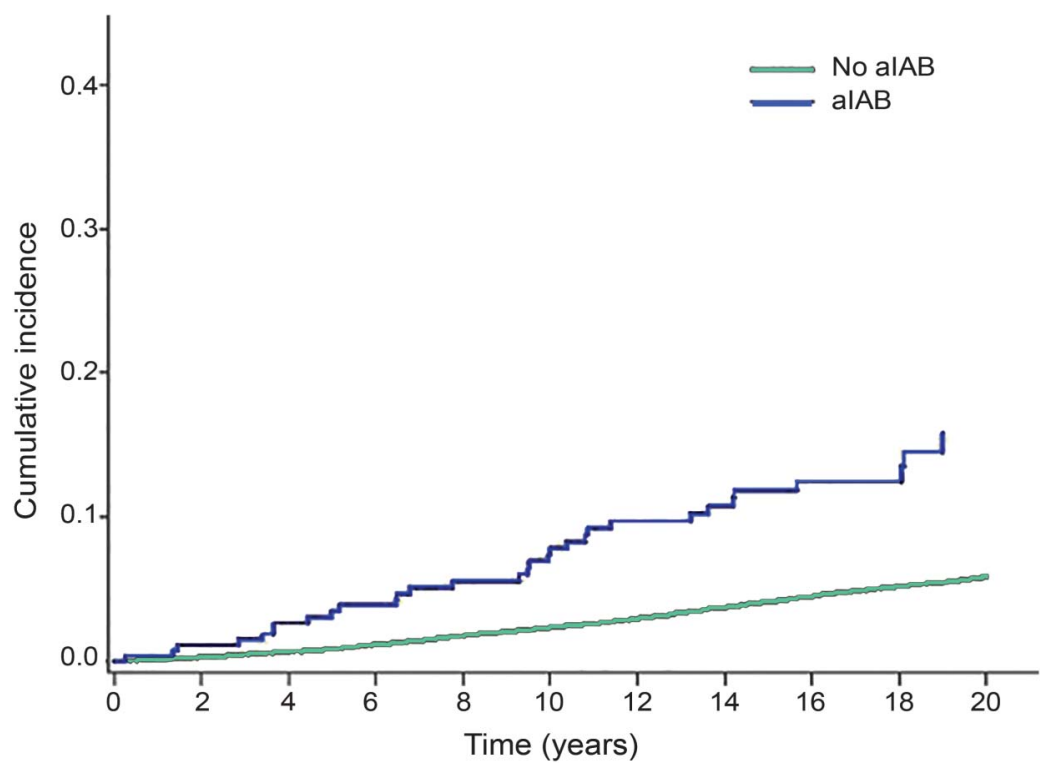

Cumulative incidence curves are statistically different $(\log -r a n k p<0.0001)$.
The cumulative incidence curves for ischemic stroke are shown in the figure (log-rank $p<0.0001)$. In a multivariable Cox regression model adjusting for stroke risk factors and potential confounders, the association between $\mathrm{aIAB}$ and ischemic stroke was statistically significant (table 2).

A total of $1,711(12 \%)$ incident AF events were detected during the study period. Adjustment for incident $\mathrm{AF}$ as a time-dependent variable did not materially alter the relationship between $\mathrm{aIAB}$ and ischemic stroke (table 2). The association between $\mathrm{aIAB}$ and ischemic stroke also was not materially altered after further adjustment for $\mathrm{PTFV}_{1}$ (HR $1.54,95 \%$ CI $1.07,2.22$ ) or $\mathrm{AF}$ as a time-fixed covariate (HR 1.53, 95\% CI 1.06, 2.20). Additionally, no interactions were observed when the analysis was stratified by age, sex, or race (table 3 ).

DISCUSSION In this analysis from ARIC, aIAB, a marker of atrial fibrosis and abnormal cardiac remodeling of the left atrium, was independently associated with an increased risk for ischemic stroke. The association between $\mathrm{aIAB}$ and ischemic stroke remained statistically significant after adjustment for established stroke risk factors. In aggregate, our findings strengthen the argument that left atrial disease should be considered an independent stroke risk factor.

Reports have recently shown that $\mathrm{PTFV}_{1}$, a marker of left atrial abnormality, independently predicts ischemic stroke..$^{2-4}$ Other reports also have implicated premature atrial contractions as potential risk factors for stroke. ${ }^{13,14}$ Both PTFV ${ }_{1}$ and premature atrial contractions predict stroke independent of diagnosed AF. ${ }^{4,14}$ Our results add strength to the argument that left atrial thromboembolism is able to occur independent of $\mathrm{AF}$ and extend this hypothesis to include aIAB. Although participants with aIAB and other markers of left atrial disease possibly develop subclinical AF, this report and the aforementioned studies show that an increased risk of stroke exists with these ECG findings. In this framework in which left atrial disease is associated with an increased stroke risk, anticoagulation strategies potentially are beneficial to reduce the morbidity and mortality associated with stroke. However, the findings of this analysis were unable to determine which participants with aIAB will benefit from such therapies and further studies are needed to explore the value of anticoagulation in persons with $\mathrm{AIAB}$ and other markers of left atrial disease before changes in clinical practice are recommended.

The pathophysiologic link between $\mathrm{aIAB}$ and ischemic stroke remains unclear. Patients with $\mathrm{AIAB}$ have been shown to have reduced left atrial contractility, leading to reductions in left atrial stroke volume and kinetic energy. ${ }^{15}$ The resultant dysfunctional left 
Table 2 Risk of ischemic stroke associated with advanced interatrial block (alAB) $(n=14,716)$

$\begin{array}{llllll} & \text { Events } & \text { Person-years } & \begin{array}{l}\text { Model } 1^{\mathrm{a}} \mathrm{HR} \\ (95 \% \mathrm{Cl})\end{array} & \begin{array}{l}\text { Model } 2^{\mathrm{b}} \mathrm{HR} \\ (95 \% \mathrm{Cl})\end{array} & \begin{array}{l}\text { Model } 3^{\mathrm{c}} \mathrm{HR} \\ (95 \% \mathrm{Cl})\end{array} \\ \text { No alAB } & 885 & 281,766 & 1 \text { (ref) } & 1 \text { (ref) } & 1 \text { (ref) } \\ \text { alAB } & 31 & 3,850 & 2.05(1.43,2.94) & 1.63(1.13,2.34) & 1.70(1.18,2.44)\end{array}$

Abbreviations: $\mathrm{Cl}=$ confidence interval; $\mathrm{HR}=$ hazard ratio.

${ }^{\text {a } A d j u s t e d ~ f o r ~ a g e, ~ s e x, ~ a n d ~ r a c e . ~}$

${ }^{\mathrm{b}}$ Adjusted for model 1 covariates plus smoking, systolic blood pressure, diabetes, body mass index, low-density lipoprotein cholesterol, antihypertensive medication use, coronary heart disease, and heart failure.

${ }^{\mathrm{c}}$ Adjusted for model 2 covariates plus atrial fibrillation as a time-dependent covariate.

atrium possibly leads to atrial thromboembolism. This is supported by a small chart review of 61 patients with embolic stroke which showed that nearly $80 \%$ of these patients had evidence of prolonged $\mathrm{P}$-wave duration $(\geq 110 \mathrm{~ms}) .{ }^{16}$ The abnormal conduction of aIAB also predisposes to the development of arrhythmias by modifying atrial refractory periods, ${ }^{17}$ leading to premature beats that possibly serve as inciting events for supraventricular arrhythmias. Due to recent reports that have shown an association between atrial ectopy and ischemic stroke, ${ }^{13,14}$ it is plausible that aIAB serves as the necessary substrate for atrial ectopy to lead to stroke. Additionally, aIAB has been associated with AF and it is possible that $\mathrm{AF}$ serves as an intermediate event between $\mathrm{aIAB}$ and stroke. ${ }^{18,19}$ However, the relationship between $\mathrm{aIAB}$ and stroke was not attenuated by incident $\mathrm{AF}$ events in this analysis. Nonetheless, it is possible that subclinical AF cases developed that were not detected. Combined with evidence that suggests left atrial thrombosis is possible with $\mathrm{AIAB},{ }^{15}$

\begin{tabular}{|c|c|c|c|c|c|}
\hline \multirow[t]{2}{*}{ Table 3} & \multicolumn{5}{|c|}{$\begin{array}{l}\text { Risk of ischemic stroke associated with advanced interatrial block by } \\
\text { age, sex, and race }(n=14,716)\end{array}$} \\
\hline & Events & $\begin{array}{l}\text { Model } 1^{\mathrm{a}} \mathrm{HR} \\
(95 \% \mathrm{Cl})\end{array}$ & $\begin{array}{l}\text { Model } 2^{b} \text { HR } \\
(95 \% \mathrm{Cl})\end{array}$ & $\begin{array}{l}\text { Model } 3^{\mathrm{c}} \mathrm{HR} \\
(95 \% \mathrm{Cl})\end{array}$ & p Interaction ${ }^{d}$ \\
\hline \multicolumn{6}{|l|}{ Age, y } \\
\hline$<54$ & 288 & $3.74(1.66,8.40)$ & $2.24(0.99,5.08)$ & $2.25(0.99,5.10)$ & 0.24 \\
\hline$\geq 54$ & 628 & $2.04(1.36,3.04)$ & $1.65(1.10,2.47)$ & $1.72(1.15,2.59)$ & \\
\hline \multicolumn{6}{|l|}{ Sex } \\
\hline Female & 448 & $2.13(1.17,3.89)$ & $1.73(0.95,3.17)$ & $1.75(0.96,3.21)$ & 0.85 \\
\hline Male & 468 & $1.98(1.26,3.12)$ & $1.59(1.01,2.51)$ & $1.66(1.05,2.62)$ & \\
\hline \multicolumn{6}{|l|}{ Race } \\
\hline Black & 366 & $1.74(0.89,3.39)$ & $1.46(0.75,2.85)$ & $1.49(0.76,2.91)$ & 0.45 \\
\hline White & 550 & $2.24(1.46,3.45)$ & $1.75(1.13,2.70)$ & $1.81(1.17,2.80)$ & \\
\hline
\end{tabular}

Abbreviations: $\mathrm{Cl}=$ confidence interval; $\mathrm{HR}=$ hazard ratio.

a Adjusted for age, sex, and race.

${ }^{\mathrm{b}}$ Adjusted for model 1 covariates plus smoking, systolic blood pressure, diabetes, body mass index, low-density lipoprotein cholesterol, antihypertensive medication use, coronary heart disease, and heart failure.

${ }^{\mathrm{c}}$ Adjusted for model 2 covariates plus atrial fibrillation as a time-dependent covariate.

${ }^{\mathrm{d}}$ Interactions tested using model 2. subsequent arrhythmic events may lead to arterial embolism. Furthermore, persons with aIAB were more likely to have well-known stroke risk factors (e.g., diabetes, hypertension, heart failure) that possibly increased their risk of developing stroke. Although we offer several mechanisms that explain the observed findings, further research is needed to determine the underlying mechanism that links $\mathrm{aIAB}$ with stroke.

The current study should be interpreted in the context of certain limitations. Several baseline characteristics were self-reported (e.g., smoking) and subjected our analysis to recall bias. Additionally, we attempted to determine if $\mathrm{AF}$ events attenuated the relationship between $\mathrm{aIAB}$ and stroke. However, paroxysmal AF cases potentially were missed due to the time-dependent nature of this condition, resulting in limited power to detect significant attenuation. Furthermore, although we included several covariates in our multivariable models that likely influenced the development of ischemic stroke, in methods similar to other epidemiologic studies, we acknowledge that residual confounding remains a possibility.

In this report, we found that $\mathrm{AIBB}$ is an independent risk factor for ischemic stroke in ARIC. Our findings strengthen the argument that left atrial disease possibly is an independent risk factor for stroke in which anticoagulation therapies are beneficial. Further studies are needed to explore the potential benefit of oral anticoagulation in persons with aIAB and other markers of left atrial disease detected on the routine 12-lead ECG.

\section{AUTHOR CONTRIBUTIONS}

Drs. O'Neal and Soliman had full access to all the data in the study and take responsibility for the integrity of the data and the accuracy of the data analysis. Study concept and design: Drs. O'Neal, Kamel, Zhang, Chen, Alonso, Soliman. Acquisition of data: Drs. O'Neal, Alonso, Soliman. Analysis and interpretation of data: Drs. O'Neal, Soliman. Drafting of the manuscript: Drs. O'Neal, Kamel, Zhang, Chen, Alonso, Soliman. Critical revision of the manuscript for important intellectual content: Drs. O’Neal, Kamel, Zhang, Chen, Alonso, Soliman. Statistical analysis: Drs. O'Neal, Soliman. Administrative, technical, or material support: Soliman. Study supervision: Dr. Soliman.

\section{ACKNOWLEDGMENT}

The authors thank the staff and participants of the ARIC for their contributions.

\section{STUDY FUNDING}

The Atherosclerosis Risk in Communities Study is carried out as a collaborative study supported by National Heart, Lung, and Blood Institute contracts (HHSN268201100005C, HHSN268201100006C, HHSN268201100007C, HHSN268201100008C, HHSN268201100009C, HHSN268201100010C, HHSN268201100011C, and HHSN268201100012C).

\section{DISCLOSURE}

The authors report no disclosures relevant to the manuscript. Go to Neurology.org for full disclosures.

Received November 13, 2015. Accepted in final form March 2, 2016. 


\section{REFERENCES}

1. Bayes de Luna A, Platonov P, Cosio FG, et al. Interatrial blocks: a separate entity from left atrial enlargement: a consensus report. J Electrocardiol 2012;45:445-451.

2. Kamel H, Soliman EZ, Heckbert SR, et al. P-wave morphology and the risk of incident ischemic stroke in the Multi-Ethnic Study of Atherosclerosis. Stroke 2014;45: 2786-2788.

3. Kamel H, Bartz TM, Longstreth WT Jr, et al. Association between left atrial abnormality on ECG and vascular brain injury on MRI in the Cardiovascular Health Study. Stroke 2015;46:711-716.

4. Kamel H, O’Neal WT, Okin PM, Loehr LR, Alonso A, Soliman EZ. Electrocardiographic left atrial abnormality and stroke subtype in ARIC. Ann Neurol 2015;78:670-678.

5. Kamel H, Okin PM, Longstreth WT Jr, Elkind MS, Soliman EZ. Atrial cardiopathy: a broadened concept of left atrial thromboembolism beyond atrial fibrillation. Future Cardiol 2015;11:323-331.

6. Rosamond WD, Folsom AR, Chambless LE, et al. Stroke incidence and survival among middle-aged adults: 9-year follow-up of the Atherosclerosis Risk in Communities (ARIC) cohort. Stroke 1999;30:736-743.

7. Robins M, Weinfeld FD. The National Survey of Stroke: study design and methodology. Stroke 1981;12:I7-I11.

8. Alonso A, Agarwal SK, Soliman EZ, et al. Incidence of atrial fibrillation in whites and African-Americans: the Atherosclerosis Risk in Communities (ARIC) study. Am Heart J 2009;158:111-117.

9. Soliman EZ, Prineas RJ, Case LD, Zhang ZM, Goff DC Jr. Ethnic distribution of ECG predictors of atrial fibrillation and its impact on understanding the ethnic distribution of ischemic stroke in the Atherosclerosis Risk in Communities (ARIC) study. Stroke 2009;40:1204-1211.

10. Epstein AE, Alexander JC, Gutterman DD, Maisel W, Wharton JM. Anticoagulation: American College of Chest
Physicians guidelines for the prevention and management of postoperative atrial fibrillation after cardiac surgery. Chest 2005;128:24S-27S.

11. Loehr LR, Rosamond WD, Chang PP, Folsom AR, Chambless LE. Heart failure incidence and survival (from the Atherosclerosis Risk in Communities study). Am J Cardiol 2008;101:1016-1022.

12. Healey JS, Connolly SJ, Gold MR, et al. Subclinical atrial fibrillation and the risk of stroke. N Engl J Med 2012;366: 120-129.

13. Binici Z, Intzilakis T, Nielsen OW, Kober L, Sajadieh A. Excessive supraventricular ectopic activity and increased risk of atrial fibrillation and stroke. Circulation 2010; 121:1904-1911.

14. Larsen BS, Kumarathurai P, Falkenberg J, Nielsen OW, Sajadieh A. Excessive atrial ectopy, short atrial runs increase the risk of stroke beyond incident atrial fibrillation. J Am Coll Cardiol 2015;66:232-241.

15. Goyal SB, Spodick DH. Electromechanical dysfunction of the left atrium associated with interatrial block. Am Heart J 2001;142:823-827.

16. Lorbar M, Levrault R, Phadke JG, Spodick DH. Interatrial block as a predictor of embolic stroke. Am J Cardiol 2005;95:667-668.

17. Daubert JC, Pavin D, Jauvert G, Mabo P. Intra- and interatrial conduction delay: implications for cardiac pacing. Pacing Clin Electrophysiol 2004;27:507-525.

18. Enriquez A, Sarrias A, Villuendas R, et al. New-onset atrial fibrillation after cavotricuspid isthmus ablation: identification of advanced interatrial block is key. Europace 2015; 17:1289-1293.

19. Sadiq Ali F, Enriquez A, Conde D, et al. Advanced interatrial block predicts New onset atrial fibrillation in patients with Severe heart failure and cardiac Resynchronization Therapy. Ann Noninvasive Electrocardiol 2015; 20:586-591.

\section{Look What's New at the 2016 AAN Fall Conference}

Visit AAN.com/view/fall to register for the 2016 AAN Fall Conference, set for October 14-16, at The Cosmopolitan of Las Vegas. This is your year-end destination for acquiring the latest clinical advances in key disease states, improving your practice's efficiency and bottom line, and earning up to $15.75 \mathrm{CME}$ credits.

\section{Look What's New!}

- All-inclusive registration rate offers greater value than ever

- Flexible meeting format lets you build your own tailored schedule and select the programs of most interest-while on the go on-site

- New Topics: Update in Stroke, AAN Leadership University Course: Challenges of Leadership in Private Practice, Headache Skills Workshop (pre-registration required) 